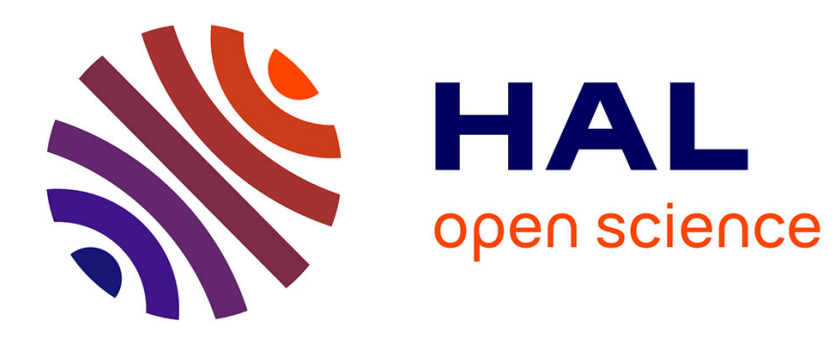

\title{
De Jericho au bang sonique. Les précurseurs des arts et sciences acoustiques \\ P. Lienard
}

\section{To cite this version:}

P. Lienard. De Jericho au bang sonique. Les précurseurs des arts et sciences acoustiques. Journal de Physique IV Proceedings, 1994, 04 (C5), pp.C5-21-C5-29. 10.1051/jp4:1994503 • jpa-00252765

\section{HAL Id: jpa-00252765 https://hal.science/jpa-00252765}

Submitted on 1 Jan 1994

HAL is a multi-disciplinary open access archive for the deposit and dissemination of scientific research documents, whether they are published or not. The documents may come from teaching and research institutions in France or abroad, or from public or private research centers.
L'archive ouverte pluridisciplinaire HAL, est destinée au dépôt et à la diffusion de documents scientifiques de niveau recherche, publiés ou non, émanant des établissements d'enseignement et de recherche français ou étrangers, des laboratoires publics ou privés. 


\title{
De Jericho au bang sonique. Les précurseurs des arts et sciences acoustiques
}

\author{
P. LIENARD
}

36 rue Gustave Vatonne, 91190 Gif-sur-Yvette, France

\begin{abstract}
After Adam, Caïn, Hénoch,... "Yubal was the father of all which as handle the harp and organ" (Bible, Genesis, IV. 21). From the Bible, and through Babylonian, Chinese, Egyptian, Arabs civilisations, the Middle-ages and after, we found many precursive ideas or realisations of our acoustic theories or instruments. Some examples are given: musical scales, vibrating wires and pipes, nature of sound, its propagation and velocity, echo and thunder, sound recording, room acoustics, sound detection, noise annoyance, even trigonometric series and Fourier Transform, shock waves and sonic boom.
\end{abstract}

\section{1 - Traces anciennes et recherches d'antériorités de l'acoustique. \\ "Il est exceptionnel qu'un chercheur puisse être absolument original, et une découverte sans signes prémonitoires" (Jean Jacques, 1987).}

Parmi toutes les sciences et inventions, l'acoustique est celle qui a laissé le moins de traces.

D'après sa racine grecque (Akustike = écouter), l'acoustique est "la science qui traite des propriétés, de la production, de la propagation et de la réception des sons" (Larousse). Or le son est un élément fugitif, qui s'évanouit dès qu'on cesse de le produire. Un écho, ou la réverbération d'une salle vide, ne lui donnent qu'un sursis de quelques secondes au plus.

Les civilisations anciennes nous ont laissé, par peinture ou graphismes, des souvenirs sur leurs connaissances et leurs talents musicaux. En explorant ce que les historiens ont pu écrire, en fouillant les archives et les musées, on voit que les hommes, cherchant à expliquer les phénomènes de la nature qu'ils entendaient, à s'exprimer par la parole, le chant et la musique, à transmettre les sons ou les conserver, ou parfois à se protéger du bruit, ont, depuis la plus haute antiquité, des notions, des connaissances, des techniques, portant les germes ou premières approximations, de notre "acoustique moderne".

Nous n'en donnerons ici que quelques exemples.

\section{2 - La nuit des temps et des sons. Premières manifestations sonores. \\ "Viracocha forma l'homme avec l'argile de la terre. Il lui donna la parole et la chanson" (Mythologie Inca).}

La création du monde mit en oeuvre les diverses énergies que connait la Physique, agissant sur la matière plus ou moins élastique, où peuvent se propager des ondes.

Adam, hors du Paradis, doué de parole et d'habileté manuelle, commence la conquête de la nature. A la sixième génération, Yubal, descendant de Caïn, "fut l'ancêtre de tous ceux qui jouent de la lyre et du chalumeau" (Bible, Genèse). 
D'après les Grecs, c'est Pan, dieu des bergers d'Arcadie, qui a inventé la flûte portant son nom; or le chalumeau de Yubal n'a qu'un seul tube, la flûte de Pan en a plusieurs de longueurs différentes et juxtaposés. L'un et l'autre personnage, probablement, ont soufflé par hasard sur le bout d'un roseau, et ont perçu un son, puis ont appris, par des moyens différents, à le moduler. Il n'y aura donc pas de procès d'antériorité entre ces deux inventeurs, Pan ayant apporté un perfectionnement au "brevet" de Yubal!

Celui-ci a d'ailleurs un deuxième brevet d'invention à son actif: les instruments à cordes. Il est probable que ce fut en pinçant une liane tendue, ou un boyau séché d'animal, sur un arc de chasseur, que Yubal perçut un son. Les mérites d'invention de Pan ne sont pas grands: un dieu est par définition très intelligent. Mais Yubal, simple mortel, a eu le mérite de remarquer des phénomènes, de les observer, et de les utiliser en contrôlant leurs paramètres: longueur du tuyau, trous, tensions des cordes... Que Yubal ait vraiment existé, ou qu'il symbolise toute une lignée, peu importe: l'acoustique, science et art, était inventée.

Il est possible d'imaginer, à partir de notre expérience actuelle, "le monde des sons" préhistorique. L'homme -pas encore "sapiens"- et l'animal vivent dans un environnement, un "paysage sonore", comme on dit actuellement, produit par les éléments naturels: vent, orage, pluie, et par les animaux eux-mêmes (cris, chants) dont l'homme ne se distingue guère.

L'existence des outils et la transmission d'une technique de taille et d'emploi, impliquent l'usage de la parole. L'évolution des formes et volumes crâniens des hommes préhistoriques montre la possibilité d'y loger des muscles vocaux, des cavités résonantes, permettant l'émission des sons requis pour un langage articulé. L'homme apprend à moduler sa voix: il chante. Les "inventions" de Yubal -ou de Pan- lui apprennent à moduler aussi les bruits; la musique, utilisée pour exprimer des sentiments, honorer des divinités, rythmer la marche, soutenir l'ardeur au travail ou au combat, sera, longtemps, une branche importante des "sciences" mathématiques ou physiques, et "la science des sciences".

\section{3 - Rois Mages, Mandarins et Pharaons}

"Dans le Panthéon hindou, Shiva est responsable de l'univers.D'une main il porte la Flamme; de l'autre la Musique. Ce sont les deux pôles du Cosmos. A l'origine est le règne absolu de la Flamme...La matière s'éveille et s'organise. La flamme fait place à la Musique".

(H. REEVES, 1989)

Le mot "musique", au sens de sons coordonnés et organisés par l'homme ou par les dieux, peut le premier nous introduire dans la recherche bibliographique de ce que furent les "soucis" acoustiques des Mages, des Mandarins ou des Pharaons. Mais les ancêtres des Mages avaient eu, déjà, des ennuis avec "l'environnement sonore"...

En Mésopotamie, les dieux font exécuter par les Hommes les durs travaux de canalisations des fleuves et d'irrigation. Mais les hommes crient (ils font même "grève"!), les chantiers sont bruyants, le bruit "empêche les dieux de dormir" et dans leur colère, ils détruisent l'humanité par le Déluge (Gilgamesh; Trad. J. Bottero).

Les textes retrouvés dans les ruines d'Ur, de Babylone ou Ninive, décrivent des instruments de musique, des règles d'harmonies (longueurs de cordes ou de tuyaux et leurs rapports) qui conditionnent l'harmonie dans ce monde ou dans l'autre, d'où le caractère sacré de la musique.

La Mésopotamie, la Chine et l'Egypte, sont en concurrence pour l'ancienneté des arts et techniques.

Avant Pythagore, avant l'Egypte, les Chinois avaient défini une gamme: "Vers 2697 avant notre ère (nombre probablement mythique...), Lin Len détermine les douze "Lu", les douze demi-tons de l'octave, chaque " $\mathrm{Lu}$ "étant lourd de signification symbolique, lié aux voyelles, couleurs, matières, éléments, points cardinaux, saisons, animaux, instruments de musique, suivant la logique de l'analogie" (Collaert, Bosquet, 1979).

Ces intervalles égaux (en échelle logarithmique), donc de rapport $\sqrt[12]{2}$, permettent n'importe quelle transpostition d'un douzième d'octave.

Ces douze " $L u^{\prime \prime}$ en relation avec les 5 humeurs et les 7 planètes, furent établis par les Chinois à partir de "la progression triple de douze termes" d'après le Père Amiot, c'est-à-dire la suite des nombres $3^{\text {n }}$, de $\mathrm{n}=1$ à $\mathrm{n}=12$; ce dernier terme, $3^{12}$, est très voisin de $2^{19}$, donc la succession de douze quintes (rapport de fréquences $(3 / 2)^{12}$ est presque égale à celle de sept octaves $(27)$; la différence est le rapport $(3 / 2)^{12 / 2}=$ $1 / 0,98664$, qui est le comma pythagoricien.

4000 ans avant notre ère, le Prince chinois Tsai Lu a calculé la racine douzième de 2 avec 24 décimales, dont les 9 premières sont exactes d'après nos ordinateurs. Mais on ne connait pas sa méthode...

Cette progression par quintes croissantes et octaves décroissantes formera, seize siècles plus tard, la gamme de Pythagore des Grecs, et sert actuellement aux accordeurs de pianos. 
L'acoustique chinoise part du concept d'ondes plutôt que d'atomes. Le monde est commandé par deux forces fondamentales: Yin, femelle, doux, et Yang, mâle, dur, qui se "répondent" et s'opposent. Quand le Yang passe par un maximum, le Yin s'annule et réciproquement. Une théorie ondulatoire est aussi "liée à l'éternel flux et reflux des deux principes fondamentaux: leYang et le Yin" (Needham, 1962).

Cet auteur compare cette alternance à celle des fonctions sinus et cosinus, impaire et paire, constamment "en quadrature"... Cependant, les deux s'intègrent comme les sons de chaque instrument dans un orchestre, comme les mouvements vibratoires qui échangent de l'énergie par un mouvement pulsé, comme l'alternance jour-nuit, soleil-lune.

Le rôle mystique et sacré de la musique, dont la justesse garantit la stabilité de l'Etat, conduisit les Chinois à la perfection de leur gamme, et à la construction de nombreux instruments, donnant les sons "de la peau, de la pierre, du métal, de la terre cuite, de la soie, du bois, du bambou, de la calebasse" (Amiot), et les sons de référence, par le Lu fondamental: tube de bambou dont la longueur (étalon) et le diamètre sont bien précis (car les Chinois ont déjà la notion de "correction de bouche" des tuyaux) et dont le contenu était aussi l'étalon de volume; et par la "cloche jaune", de dimensions très précises et d'un alliage bien défini.

Un essai fut fait aussi par les Chinois d'une gamme bien tempérée, ou des approximations permettent de rapprocher certains intervalles des "rapports agréables à l'oreille" -dira Pythagore- des tierces $(6 / 5)$, quartes $(4 / 3)$, quintes $(3 / 2)$, que ne donne pas exactement la suite des racines $n / 12$ de $2 \ldots$ tentative d'harmonie subjective bien réussie plus tard par J.S. Bach...

Près des Ziggourats comme des Pyramides ou des Obélisques, du Temple de Jérusalem ou du Parthénon, le "professeur d'acoustique" fut toujours un haut personnage, Prince, Roi ou Dieu.

Chez Pharaon, les musiciens étaient des "fonctionnaires" formant une véritable aristocratie, dans laquelle, dès la fin de la Quatrième Dynastie (vers 2500 avant J.C., époque des grandes Pyramides) une hiérarchie élevait le chef des chanteurs et flûtistes de Pharaon "au-dessus de la masse des exécutants" (H. Hickman, 1554). Le musicien avait droit, pour sa sépulture, à un "mastaba" (monument dont la forme la plus gigantesque est la pyramide) dans la nécropole de Saqqarah.

Les instruments de musique, percussions ou sistres (rondelles de métal enfilées sur une tige) rythmaient la danse et la marche (ou le travail forcé...). La flûte de roseau est un instrument international, et deviendra Aulos grec, aubois, orgue (hydraulique à Alexandrie) jusqu'à nos instruments; les cordes de luth s'étendront en harpes, cithares, violles... que les Hébreux découvriront dans leur déportation en Egypte, et adapteront pour soutenir la ferveur des cérémonies religieuses, fêtes et deuils.

Mais, comme les dieux de l'Euphrate, Pharaon désirait parfois le silence: "L'Ile Pure (Bigen, près d'Assouan) devait demeurer dans le silence pour que le repos du dieu ne fut pas troublé. Il était interdit d'y frapper le tambourin... On ne devait pas y chanter, ni jouer flûte ou harpe..." (F. Daumas, 1980).

Dans les "curiosités acoustiques" égyptiennes, notons la statue de Memnon, fils de l'Aurore, qui chantait chaque matin pour saluer sa Mère. Glissement des strates de pierre dilatées par le soleil, comme les rochers du Hoggart, ou les couches de sel d'un rivage de la Mer Caspienne, qui crépitent en se réchauffant après l'humidité de la nuit, ou bruits aérodynamiques dûs au vent dans les fissures de la pierre, maintenant bouchées par la restauration de Septime Sevère?...

Conservons-en le charme mystique, et laissons aux Philosophes et aux "Positivistes" le soin d'étudier, en fait, qu'est-ce que ce son, "parvenant jusqu'à l'âme..."?

\section{4 - Qu'est-ce que le Son?}

"Nous pouvons définir le son comme un coup donné par l'air à
travers les oreilles, au cerveau et au sang et arrivant jusqu'à l'âme. Le
mouvement qui s'en suit, lequel commence à la tête et se termine dans
la région du foie, est l'oü̈e. Ce mouvement est-il rapide, le son est
aigu; s'il est plus lent, le son est plus grave; s'il est uniforme, le son
est égal et doux; il est rude dans le cas contraire; il est fort grand,
lorsque le mouvement est grand, et faible s'il est petit" (Platon,
Dialogue. Timée, vers 300 avant J.C.).

Le "philosophe", ami de la Sagesse, désire connaître les lois de la nature et y conformer sa vie.

Il fait des observations, et sa raison le conduit à "ramener à des causes simples et générales l'infinie variété des formes et des phénomènes. Ce qui caractérise la science de l'antiquité, c'est la faiblesse de la base expérimentale et la luxuriante abondance des hypothèses et des théories générales" (Cogniot, 1974).

Premier "Sage", géomètre et musicien, Pythagore nous est connu par sa Table (de multiplication), son Théorème (du carré de l'hypothénuse), et sa Gamme. Personnage légendaire, mage ou "homme divin" (on le disait fils d' Apollon), Pythagore, né dans la première moitié du 6ème siècle avant J.C., dans l'Lle de Samos, n'a pas écrit, mais enseigné verbalement, et inventé le mot de "Philosophe". Les "Acoustiques" étaient les élèves "auditeurs", qui n'avaient que le droit d'écouter. 
Les Pythagoriciens ne distinguent pas l'arithmétique, la géométrie, la physique (dont la musique et l'acoustique), ces connaissances étant toutes référées aux nombres et à leurs rapports, "élevant l'arithmétique au dessus des besoins des marchands"; mais le nombre pythagoricien devient "le principe, la source et la racine de toutes choses".

La musique devant conduire à l'harmonie des esprits est conditionnée par les nombres et les rapports simples des mathématiciens, qui sont, nous le savons maintenant, des rapports de fréquences.

Les Anciens n'ont pas encore explicité cette notion de fréquence; elle apparait cependant, dans la pensée chinoise des flux et reflux de Yin et de Yang, et leurs oscillations.

On peut interpréter la pensée de Platon, si les "mouvements rapides ou lents" sont attribués à la vitesse vibratoire, et aux "mouvements des corps sonores" déjà entrevus par Aristote.

Pour Lucrèce (De Natura Rerum, premier siècle avant J.C.), ce sont les "atomes" plus ou moins lisses ou rugueux, lents ou rapides, qui fixent les qualités des sons, première ébauche d'une "analyse spectrale".

"Ne vas pas croire que l'aigre grincement de la scie stridente soit fait d'atomes aussi lisses que les musiques mélodieuses qu'éveillent et modulent sur la lyre les doigts prompts des musiciens... Toute figure qui plait aux sons comporte des éléments lisses" (De Natura Rerum).

En remplaçant le caractère régulier des "atomes" par celui du spectre sonore, on aurait une image assez juste des sons musicaux opposés aux sons aléatoires ou dissonants.

Selon Aristote, les "corps simples" se meuvent suivant les modèles géométriques de deux figures simples: "translation linéaire" et "translation circulaire" que nous appellons rotation.

"Il est de toute nécessité qu'il existe un corps simple dont la nature soit de se mouvoir suivant la translation circulaire, conformément à sa propre nature. Le cercle entre dans la classe des choses parfaites" (Aristote).

Représenter tous les mouvements des "corps simples" par des mouvements circulaires évoque pour le physicien les séries de fonctions circulaires de Bernoulli (1753) et la transformation de Fourier (18071922).

Mais le son est-il seulement un mouvement d'atomes ou le déplacement de "simulacres" reproduisant la surface des choses... et qui ressemblent bien à nos surfaces d'ondes, de Huygens et Fresnel, "... pellicules détachées de la surface des objets, elles voltigent... Chacune a la forme et l'aspect de l'objet qui l'a émise pour la laisser vagabonder dans l'espace" (Lucrèce).

Au 17ème siècle, Galilée, dit-on, observa les oscillations d'un lustre de la Cathédrale de Pise, puis remarqua, en gravant une plaque de métal, que si l'outil grinçait, il laissait des rayures équidistantes, d'autant plus serrées que le son était plus aigu, et exactement deux fois plus, si le son passait à l'octave: première notion précise de fréquence et longueur d'onde.

Déjà, des paramètres plus "objectifs" que ceux de la musique, élargissaient le domaine des sons: "J'ai donc cru qu'il y avait une science supérieure à la musique, que j'ai appelée "ACOUSTIQUE", qui a pour objet le Son en général, au lieu que la musique a pour objet le Son en tant qu'il est agréable à l'oüe" (Sauveur, 1701).

Il faut passer au 19 ème siècle pour voir les premiers enregistrements sur papier enfumé par Young (1807), le "Phonotaugraphe" de Scott et Koenig (1861), la capsule de Marey (1872) et les oscillations des flammes de gaz sur les résonateurs d'Helmhotz, rendant visibles les formes d'ondes sonores, et contenant en germe l'enregistrement sonore qui sera décrit par Charles Cros et réalisé par Edison, qui n'a rien écrit, mais a fait.

Atomes ou simulacres se déplacent, mais pas instantanément. (Une vitesse infinie serait contraire à la doctrine d'Aristote).

\section{5 - Zeus, son Epouse et les Nymphes. \\ Deux mystères: l'Echo et le Tonnerre.}

"Ekho, fille de Junon, ... nymphe meurtrière, a des intelligences partout... dans les bois, rus de rivières, marais, continuations de murailles... pressoirs etc..." (Mersenne, 1634).

Ekho, nymphe des montagnes, des sources et des forêts, avait tous les charmes de son état, mais un défaut irritant: elle était bavarde! Elle essaya, par des flots de paroles, de distraire l'attention d'Héra, déesse jalouse et vindicative, qui surveillait les infidélités de Zeus, son fulgurant époux, trop entouré des séduisantes compagnes d'Ekho. Vexée, Héra punit celle-ci en lui ôtant l'usage de la parole, ne lui laissant que la faculté de répéter les dernières syllabes de son interlocuteur (Ovide).

Une voix inconnue, incompréhensible, venue on ne sait d'où, était propre à intriguer, inquiéter ou effrayer ses auditeurs. Il y avait là, caché dans la montagne, le mur ou la forêt, un être puissant, qui répondait, et qu'il serait incorrect, voir dangereux, de mépriser. 
Averroes, savant arabe né à Cordoue, qui a importé en Europe la doctrine d'Aristote, vers 1200 , "nous représente la nature de l'écho comme les cercles qui sont produits en l'eau par le moyen d'une petite pierre... ayant rencontré le bord, les cercles retournent vers l'endroit d'où ils sont partis... Aristote a enseigné en son second Livre de l'Ame, comme une balle poussée contre un corps solide revient du côté d'où elle est partie... De cette collision provient le son" (Mersenne, 1634).

Montesquieu, en 1718, obtint un prix de l'Académie de Bordeaux pour une étude sur les ondes sonores et l'écho, qu'il considère comme une cause du tonnerre (texte de 1726, semblant perdu), ce que d'Alembert résume dans l'Encyclopédie (1735, article Echo): "son réfléchi ou renvoyé par un corps solide et qui par là se répète et se renouvelle à l'oreille".

Les descriptions d'ondes par Huygens et Fresnel expliquent parfaitement tous les phénomènes d'écho observés en montagne ou en de nombreux bâtiments.

Combien plus que l'écho, les manifestations du tonnerre, "son et lumière", ne devaient-elles pas inspirer la crainte d'une puissance vénérable, Zeus ou Jupiter, régnant dans les cieux.

Epicure, Lucrèce et Aristophane n'ont pas besoin de l'hypothèse Zeus, mais "les nuages en ce choquant ont fait jaillir de nombreux atomes ignés..." ce qui n'est pas si faux; et Aristophane, vers 400 avant J.C. dans sa comédie "Les Nuées", dit à son disciple que "les nuées, en se roulant, crèvent avec fracas et tonnent grandement... à l'échelle de l'air sans limite..." par rapport "à ton petit ventre où tu entends des troubles et des crépitements" après les festins!

L'hypothèse d'une réverbération sur les nuages du bruit très court de l'éclair est proposée par l'Abbé Nollet, expérimentateur de l'électrostatique: "Le tonnerre pourrait n'être bien que l'électricité" (1743), théorie que Monge réfute en 1790: "On a toujours regardé le bruit du tonnerre comme celui que devrait naturellement produire une décharge électrique aussi forte, et cette erreur a empêché de faire attention à des circonstances qu'il était cependant nécessaire de connaître pour expliquer le phénomène."

"On a cru l'expliquer en considérant le roulement du tonnerre comme produit par des échos multipliés sur les nuages... Les nuages n'ont pas la faculté de réfléchir les sons"... et il crut observer des compressions et détentes successives de petits nuages après l'éclair.

"Monge ne pouvait avoir aucune connaissance d'une multitude de détails que le progrès des sciences a rendu familiers même aux élèves de nos écoles" (Arago, 1846).

Mais, après observation des sons diffusés par les nuages dans les expériences de propagation du son entre la Tour de Montlhéry, Villejuif et Montmartre: "Ne sera-t-il pas permis de conclure de là qu'à Villejuif les coups multiples du canon de Montlhéry résultaient d'échos formés dans les nuages, et de tirer de ce fait un argument favorable à l'explication qu'ont donnée quelques physiciens du roulement du tonnerre?" (Arago, 1822).

La réflexion ou "rétrodiffusion" des ondes sonores par les couches atmosphériques de températures différentes ou par les couches nuageuses est maintenant bien connue et utilisée par le Sodar pour sonder l'atmosphère, comme le Sonar explore le fond des mers, car maintenant nous connaissons bien cette mystérieuse vitesse ou "célérité" du son, ondes ou corpuscules qui se transmettent leur mouvement.

\section{6 - Une autre inconnue: la célérité du son.}

"Si toutefois le bruit du tonnerre n'est perçu par nos oreilles qu'après l'apparition de l'éclair à nos yeux, c'est que les sons cheminent toujours plus lentement que les éléments destinés à émouvoir la vision. Tu pourras toi-même t'en rendre compte en regardant de loin le bucheron tailler de sa hache... un arbre...; tu apercevras le coup avant que le bruit du choc ne retentisse à ton oreille" (Lucrèce de Natura Rerum, 95 avant J.C.).

Mersenne, en 1636, et Gassendi, entreprirent des expériences systématiques: cette célérité varie-telle avec la hauteur tonale du son (d'après Aristote, les sons aigus sont plus rapides) et son intensité? Estelle affectée par le vent? Les expériences utilisaient un canon, un mousquet ou un pétard, et on comptait les oscillations d'un pendule entre l'observation de la lueur et celle de la détonation. "Notre ami Mersenne... $a$ étudié avec soin la vélocité du son, et apprit qu'il couvrait en une seconde deux cent trente toises de Paris..." (Gassendi, 1658).

Comparant la vitesse de sons très différents en tonalité et intensité, Gassendi montre que celle-là ne dépend pas de la fréquence et de l'intensité (dans le cas, le plus fréquent, de ce que nous appellons "l'acoustique linéaire").

La vitesse du son et ce principe de mesure furent l'un des thèmes du Dialogue de Galilée:

"-Simplicio: ... la propagation de la lumière est instantanée: quand nous voyons de loin une pièce d'artillerie, l'éclat de la flamme, sans interruption de temps, se porte à nos yeux, tandis que le son ne parvient à nos oreilles qu'après un intervalle de temps appréciable. 
-Sagredo: Hélas, nous ne sommes pas assurés pour cela que la vitesse de la lumière soit instantanée plutôt que très rapide!"

De nombreux expérimentateurs ont donné des résultats numériques assez divergents, surtout en raison de la diversité des mesures de longueur utilisées: 1077 pieds par seconde, ou 230 toises de 6 pieds, ou 180 toises, ou 1080 pieds... Pieds de Roy, pieds anglais, toises de Paris... et Euler, Suisse, ajoute des mesures en pieds du Rhin...

L'Académie Royale des Sciences de Paris décide en 1738 de lancer une série d'expériences rigoureusement conduites, afin d'obtenir une valeur plus précise de cette vitesse du son, et de ses variations: "Il ne parait pas qu'on ait encore déterminé avec toute la précision requise l'espace qu'il parcourt dans un temps donné, et qu'on ait fait toutes les expériences nécessaires pour constater si la vitesse est toujours uniforme dans les plus petites comme les plus grandes distances, et s'il y arrive des variétés suivant les différentes circonstances des temps, du jour et de la nuit, du temps serein ou de la pluie, de la force et de la direction du vent" (Cassini, 1738).

La Commission nommée par l'Académie était conduite par ce géomètre, d'une famille qui, de père en fils, avait relevé la "Carte de Cassini", modèle de nos cartes I.G.N. Les bases de mesure furent prises dans les triangles de cette cartographie, principalement de la Tour de Montlhéry à la pyramide đu méridien de Paris, à Montmartre $(29,6 \mathrm{~km})$.

On "tirait le canon" en l'un ou l'autre point, et le temps était mesuré par un pendule et une "montre à seconde" (à $1 / 2$ seconde près). Les expérimentateurs notaient aussi la température et le sens du vent (ceci éliminé par les deux expériences en sens contraires). De nombreuses difficultés apparurent: mauvais temps, bruit parfois inaudible, ou au contraire, réception de plusieurs échos, vent variable, ou... trop grande affluence de curieux, bruyants...

Quelles que soient les circonstances, la moyenne des mesures conduisit à une valeur de 173 toises par seconde. L'effet du vent était encore mal précisé: "Le Père Mersenne et Gassendi ont assuré que le vent, favorable ou contraire, n'accélérait ni ne retardait le son; depuis les expériences que Derham et l'Académie des Sciences ont faites sur ce sujet, cela passe pour une erreur" (Encyclopédie, Article "Son", 1765).

C'est encore à un géomètre et explorateur, La Condamine, que l'Académie confie la mission de mesurer des arcs de méridien en Amérique, et à cette occasion, la vitesse du son entre Cayenne et Kourou (maintenant bien connue pour ses activités spatiales). Il y découvrit aussi l'arbre dit "Cahuchu" dont la sève est utilisée par les Indiens.

Suivant les mêmes méthodes que Cassini, il trouve une vitesse du son de 183,5 toises en ces lieux (1739), soit $357 \mathrm{~m} / \mathrm{s}$. Il y faisait nettement plus chaud qu'à Montlhéry en Mars 1738! (Ces vitesses conduisent, suivant les formules actuelles de la vitesse du son, à des températures de $10^{\circ} \mathrm{C}$ à Montlhéry en Mars et $44^{\circ} \mathrm{C}$ à Kourou en été).

Bien d'autres mesures furent faites ensuite, soit à l'air libre, soit dans des tuyaux (Regnault, Violle et Vauthier) avec des membranes et des contacts électriques pour préciser l'instant de passage des ondes.

Mais l'écart des résultats expérimentaux persistait avec les calculs de Newton, conduisant à une valeur de c toujours inférieure de l'ordre de 20\%. Et parmi les "géomètres" (Lagrange, Euler, Poisson, Laplace) "personne n'a bien trouvé la cause de cette accélération sur la théorie" (Euler, 1759).

On sait que l'erreur n'était pas dans le calcul de Newton, mais dans son hypothèse de départ; compression isotherme du gaz dans les ondes sonores, et non pas adiabatique.

La prise en compte dans le calcul, par Lagrange(1759) puis Poisson (1808) de l'élasticité

volumique, introduit l'exposant $\gamma$; la vitesse du son devint $\sqrt{\gamma p / \rho}$, au lieu de $\sqrt{p / \rho}$ de Newton. Les travaux de Laplace, Regnault, Gay Lussac, Laroche et Bérard, Clément et Desorme, précisèrent la valeur numérique de ce coefficient: 1,4 pour l'air, dont la racine carrée est de l'ordre de 1,2, et voilà cet écart de $20 \%$ expliqué!

Laplace suggère même que la mesure de $c$ pourrait être assez précise pour conduire plus sûrement à la valeur de $\gamma$ que par mesure directe.

\section{7 - Comment le son diminue-t-il lors de sa propagation?}

"Le son diminue quand les espaces augmentent. Or la surface de ces espaces est en raison doublée -c'est-à-dire "proportionnelle au carré"-de la distance du son d'avec les corps par lesquels il a été premièrement produit, et conséquemment le son diminue en proportion géométrique... Si la lumière a 64 degrés de force à un pas ou telle distance du soleil ou de la chandelle que l'on voudra, elle sera 4 fois moins à 2 pas, 16 fois moins à 4 pas. " (Mersenne, 1633)

Mais les affaiblissements observés sont encore supérieurs à ceux dus à la simple géométrie, et Mersenne se préoccupe de cette cause, qu'on trouvera beaucoup plus tard: viscosité de l'air, échanges moléculaires... 
Les mesures d'intensité ne seront possibles qu'au 20ème siècle. Une mesure indirecte des variations d'intensité était alors faite à l'oreille, par une "méthode de zéro": par l'inverse du carré de la distance à laquelle on n'entend plus le son.

Il semble que le pasteur anglais Derham ait le premier constaté l'effet du sol sur la propagation, en observant la décroissance du son des cloches de son église, en s'en éloignant à cheval, quand la neige tombait.

Son ami Averrani et lui ont fait des observations précises, en des climats différents, et suggéré l'influence possible sur la transmission de l'intensité, des variations de température et de densité de l'atmosphère; Derham en déduisit même une méthode acoustique de "sondage" de cette atmosphère, qu'il appelle "échométrie" utilisant une "échosonde"; moyens maintenant développés dans le Sodar. Le mot "sonde", d'après Brown et Hall (1878) est une variante de "son" ("sound") pour désigner l'appareil qui perçoit ou mesure l'écho, mot qui, du temps d'Homère, désignait plus globalement le son audible.

La raréfaction de l'air, dans une cloche "à vide", ne semblait pas empêcher la propagation du son, suivant les expériences de Kircher (1650) et de Otto de Guerick (1672). Robert Boyle, en 1682, réussit enfin à faire un vide assez poussé pour empêcher la propagation du son hors de la cloche.

Dans un but d'aide à la navigation, par signaux lumineux ou sonores, Tyndall (1874) étudia la transparence optique et la transparence acoustique de l'atmosphère, au moyen d'une trompette de plusieurs mètres de longueur, mue par la pression d'une machine à vapeur, placée sur une falaise de Douvres, et dont le son était entendu par plusieurs navires en mer.

\section{8 - Le son se propage-t-il dans l'eau et dans les solides? \\ "Franklin* s'était assuré que le bruit de deux cailloux choqués dans l'eau est encore sensible à plus d'un demi-mile" (Colladon, 1827).}

Pline avait admis que "les poissons entendent". Pour vérifier l'expérience de Franklin, l'Abbé Nollet n'hésita pas à s'immerger, à une époque où les bains de rivière étaient peu usuels... surtout pour un ecclésiastique!

$\mathrm{Au} 18 \mathrm{ème} \mathrm{siècle,} \mathrm{Beudant,} \mathrm{minéralogiste,} \mathrm{fit} \mathrm{les} \mathrm{premières} \mathrm{expériences} \mathrm{quantitatives,} \mathrm{près} \mathrm{de}$ Marseille, en frappant une cloche dans l'eau; un nageur, à proximité, levait un drapeau lorsqu'il entendait. Il trouva une célérité de $1500 \mathrm{~m} / \mathrm{s}$, ce qui est remarquable avec de tels moyens!

D'après ses équations, appliquées aux milieux liquides, Laplace trouva $1525 \mathrm{~m} / \mathrm{s}$ dans l'eau de pluie et 1621 dans l'eau de mer.

Sur la proposition de l'Académie des Sciences, un jeune physicien suisse, Colladon, Docteur en droit mais passionné de physique, mesura la compressibilité de l'eau (ce qui lui valut un prix de l'Académie en 1826) et avec son ami Sturm entreprit des mesures dans le Lac Léman, avec deux bateaux, une cloche dans l'eau dont le marteau allumait en haut une charge fulgurante, et un tube plongé dans l'eau, à $50 \mathrm{~km}$ de là, recevant le signal très peu affaibli. Ils trouvèrent $1435 \mathrm{~m} / \mathrm{s}$ dans l'eau à $8^{\circ} \mathrm{C}(1827)$.

Ces observations furent à la base de la méthode actuelle de détection sous-marine et des Sonar, développée à notre époque grâce aux travaux de P. Langevin, L. Florisson, R. Lucas, P. Biquart, avec des ultra-sons, des capteurs piezoélectriques.

Mais cette idée remonte à Léonard de Vinci... "Si tu places un long tube dans l'eau... tu entendras des navires à une grande distance".

Les vibrations des solides furent étudiées par Chladni, Lissajous,... et Hassenfratz (1794), mesurant l'élasticité des roches, prélude à la prospection sismique. Un siècle plus tard, De Place présentait à l'Académie un "schiséophone" servant à explorer la nature intime des masses métalliques à l'aide d'un procédé électro-mécanique (1892).

\section{9 - Les sons en conserve.}

D'un rêve d'autrefois aux réalisations d'aujourd'hui. "Icy est le confin de la Mer Glaciale, sur laquelle feut, au commencement de l'hyver dernier passé, grosse et félonne bataille entre les Arismapiens et les Néphélibates. Lors gelèrent en l'air les paroles et crys des hommes... les hurtys des harnais... et tout autre effroy de combat. A cette heure,... advenant la sérénité et tempérie du bon temps, elles fondent et sont ouyes" (F. Rabelais, Quart Livre, 1548).

* John Franklin (1786-1847) et non Benjamin (1706-1790) inventeur du paratonnerre. 
Fixer les sons dans une matière quelconque, les entendre à nouveau, longtemps après, et autant de fois qu'on veut, est depuis toujours l'un des rêves de l'humanité.

En écoutant les sons confus, modulés, qui sortent d'une conque marine appuyée contre l'oreille sans en fermer l'orifice, les Anciens croyaient que le son de la mer s'y était enfermé.

Les paroles proférées à voix haute, les discussions et altercations pourraient-elles imprégner les murs qui en furent les témoins?

Dans les remparts de Mégare, en Grèce, construits par Apollon, une pierre était "sonore", imprégnée des sons de la lyre qu'Apollon y avait posée.

Le sol lui-même, dans la mythologie, a reçu et divulgué un secret. Le Roi Midas, ayant osé critiquer la flûte d'Apollon, fut affligé par celui-ci d'oreilles d'âne, dissimulées par un ample bonnet, et seul son esclave connaissait le secret. Obsédé par la charge de celui-ci, l'esclave creusa un trou, et lui dit: "Le Roi Midas a des oreilles d'âne."... et partit soulagé. Mais à l'été prochain, les blés, agités par le vent, répétèrent: "Le Roi Midas a des oreilles d'âne".

En 1632, un explorateur des Terres Australes dit avoir découvert dans ce pays lointain que les hommes parlaient "à de certaines éponges qui retiennent les sons et la voix comme les nôtres font des liquides... Ils les envoient à leurs correspondants, qui, en les pressant, en font sortir les paroles et les sons..."

Plus prè̀s de nous, Cyrano de Bergerac au 17ème siècle (de Bergerac sur Yvette... au château de Mauvières, près de Chevreuse...) explorant les "Empires du Soleil et de la Lune", remarqua une étrange boîte, "pleine de petits ressorts. C'est un livre, où les yeux sont inutiles, on n'a besoin que des oreilles". Il suffit de tourner une aiguille sur le chapitre qu'on veut entendre.

Cette boîte est portative, et accompagne, en marchant, ceux qui veulent s'instruire, et "en ouïr seulement un chapitre ou bien plusieurs".

"Ce présent m'occupa plus d'une heure; et enfin, me les étant attachés en forme de pendant d'oreille, je sortis me promener".

Cyrano est-il l'initiateur de la "Science-fiction" et du "Walkman"?

Scott, et d'autres, inscrivirent sur papier les oscillations des ondes sonores. Ch. Cros, photographe, eut l'idée de solidifier ces sillons par reproduction photographique, et d'animer ensuite un pavillon qui reproduirait les sons. Il l'écrivit mais ne le réalisa pas. Th. Edison n'écrivit rien... mais il le fit, avec une gravure mécanique.

Lui et d'autres fabriquèrent et vendirent Phonographe (présenté le 11 Mars 1878 à l'Académie des Sciences, ce qui provoqua des remous... accusation de supercherie!), graphophone et gramophone (Berliner et Hermann, 1888).

H. Bouasse, professeur à l'Université de Toulouse, ne sait si on doit plus admirer "leur extrème ingéniosité ou leur parfaite inutilité"!!

Utile ou pas, l'enregistrement sonore fut perfectionné par Tainter et Bell (inventeur du téléphone, en même temps que Gray... Mais celui-ci déposa son brevet 2 heures après Bell... A 2 heures près, le "décibel" se serait-il appelé "décigray" ?)

Puis l'électricité facilite le travail du burin graveur, et la lecture par le "pick-up" et son amplificateur (Brown-Marconi, 1927) améliorant considérablement la qualité sonore.

La lecture optique utilise l'effet photophonique du sélénium, suivant l'idée de Bell en 1880, et permet le cinéma sonore et parlant (le "parlant" exigeant une parfaite synchronisation son-image) sur piste optique, d'abord séparée de la bande-image (Gaumont) puis jointe à celle-ci. Philips utilise le système de gravure Miller par un burin triangulaire sur une couche de vernis noir.

Le micro-sillon de Goldmark-Columbia, et la qualité physico-chimique du disque, diminuent l'encombrement du support, augmentent la capacité, améliorent la bande passante et le rapport son/souffle.

Mais tout cela est dépassé par l'enregistrement magnétique. La possibilité de fixer sur l'acier des "vertus magnétiques" différentes en différents points, est indiquée par l'Encyclopédie en 1751 (article "Aimant") et son application à l'enregistrement sonore suggérée par un américain, Oberlin Smith, en 1888.

Dix ans après, Poulsen, technicien dépanneur de téléphone à Copenhague, retrouve ces "traces magnétiques" sur une bande d'acier, et prend un brevet de "télégraphone", pour conserver les télégrammes, utilisant un fil d'acier devant un électro-aimant, premier "répondeur téléphonique".

Poulsen et son ami Pedersen enroulent le fil en hélice sur un cylindrique (souvenir du cylindre d'Edison?) alors que le "dictaphone" le place sur des bobines.

Les premières "bobines magnétiques" commerciales, sur ruban d'acier, furent réalisées par Stille et Marconi en 1931. La bobine de Lorenz, ruban d'acier défilant à $1,5 \mathrm{~m} / \mathrm{s}$, pesait $15 \mathrm{~kg}$ pour trente minutes d'enregistrement, et l'appareil de lecture pesait $150 \mathrm{~kg}$ !

En 1937, Pfleumer réussit à fixer de la poudre d'oxyde de fer sur une bande plastique, et dans son brevet, crée le nom de Magnetophon.

Malgré les améliorations (prémagnétisation, modulation de fréquence, etc) tout cela est dépassé par l'enregistrement numérique, d'après le principe exposé en 1679 par Leibnitz: "Rechnung mit Null und 
Einz" (compter avec des Zéro et des Un) ce que permettront le rayon Laser et les ordinateurs à lecture et traitement rapides.

Inutile?... Oh, Monsieur le Professeur Bouasse!

\section{0 - Et d'autres inventions, plus récentes?}

L'acoustique architecturale eut pour ancêtres les Théâtres antiques, magnifiquement étudiés par F. Canac (Ed. CNRS 1967), les travaux de Vitruve (1er siècle avant J.C., mais édité en 1486) et les vases grecs résonateurs, redécouverts par cet auteur.

C'est maintenant l'ordinateur qui étudie les salles. Le résultat est-il meilleur?

Les applications militaires de l'acoustique furent nombreuses depuis la détection des vibrations du sol causée par le travail des sapes, ou la marche des troupes, décelées par les Oies du Capitole, comme par les Gaulois de la guerre de Trente ans, ou ceux des tranchées de $1915 \ldots$ suivant une idée qu'on trouve -encore...- dans les Carnets de Léonard de Vinci.

Lors de cette guerre, les avions allemands menaçant Paris étaient détectés par leur bruit, collecté par les grands "paraboloïdes" de R. Baillaud; il utilisait -sans le savoir...- les connaissances remontant à Aristote, Averroes, Mersenne...sur la réflexion du son et l'analogie du son et de la lumière.

A cette époque 1914-18, les obus de 75 étaient déjà supersoniques, à $\mathrm{M}=2$; les "ondes de choc" des mobiles supersoniques ont été montrées d'abord par le Capitaine De Labouret en 1888, puis par Ernst Mach en 1890. En 1907, un anglais, Mallock, expliqua aux artilleurs la différence entre l'onde de bouche du canon et "l'onde de choc" du projectile (1908), étude complétée de 1915 à 1918 par "l'Acoustique des canons et des projectiles" de E. Esclangon, restée secrète, puis publiée en 1925, où l'on trouve toutes les explications des "bangs soniques" et "double bangs" de nos avions modernes, si surprenants dans les années $60 . .$.

Mais des observations analogues ont déjà été dessinées par Huygens en 1680, par un "comptegouttes" se déplaçant au dessus d'une surface d'eau, à vitesse plus grande que les ondes capillaires...

Nul novi sub Sole?

BIBLIOGRAPHIE SOMMAIRE. Ouvrages généraux

DAUMAS M.

DAUMAS M. HUNT F.

LIENARD P.

LINDSAY B.

MILLER O.C.

SERRES $M$.

TATON R.
Histoire générale des techniques. (5 vol.) PUF 1952-68.

Histoire de la Science. Gallimard 1952.

Origins in Acoustics. The science of sound from antiquity to the age of Newton.

Yale Univ. Press 1978. Rééd. Acous. Soc of Amer.1992.

De Jéricho au Bang sonique. Petites histoires de l'acoustique (en préparation).

The story of acoustics. Jour.ac. Soc of Amer. 89- 4.1966.

Anecdotal history of the science of sound, to the beginning of the 20th Century. Mc Millan 1935.

Eléments d'histoire des sciences. Bordas 1989.

Histoire générale des sciences (5 vol:) PUF 1957-66. 